\title{
Gender differences of the improvement in balance control based on the real-time visual feedback system with smart wearable devices
}

\author{
I-LIN WANG ${ }^{1}$, Li-I WANG ${ }^{2}$, SHI-JIE XUE ${ }^{3}$, RUI HU ${ }^{3}$, RONG-JiUn JIAN ${ }^{4}$, CHUN-SHENG Ho ${ }^{4,5 *}$ \\ ${ }^{1}$ College of Physical Education, Hubei Normal University, Huangshi, Hubei, China. \\ ${ }^{2}$ National Dong Hwa University, Hualien, Taiwan. \\ ${ }^{3}$ Graduate Institute, Jilin Sport University, Jilin, China. \\ ${ }^{4}$ Lo-Hsu Medical Foundation, Inc., Lotung Poh-Ai Hospital, Luodong, Taiwan. \\ ${ }^{5}$ Department of Physical Therapy, College of Medical and Health Science, Asia University, Taichung, Taiwan.
}

\begin{abstract}
Purpose: The body maintains stability by integrating inputs from the central nervous system of vision, hearing, proprioception, and multiple senses. With the development of smart wearable devices, smart wearable devices can provide real-time center of pressure position-assisted balance control, which is beneficial to maintain physical balance. Methods: Forty healthy college students (20 male, 20 female) participated in this study, and the posture balance actions of left-leg stance non-visual feedback, left-leg stance visual feedback, right-leg stance non-visual feedback, and right-leg stance visual feedback were performed. Visual feedback provided smart insoles matching Podoon APP on a tablet computer with the COP position displayed by a dot as real-time visual feedback. Results: The experimental results show that the displacement, velocity, radius, and area of the COP decreased significantly in the left-leg stance visual feedback/right-leg stance visual feedback, the test compared the parameters in the left-leg stance non-visual feedback/right-leg stance nonvisual feedback $(P<0.05)$. Providing visual feedback through intelligent insoles can reduce the movement of the center of mass and maintain physical stability for healthy young people of different genders. In the one leg visual/non-visual in standing, the COP maximum anteroposterior displacement, COP anteroposterior velocity, COP radius, and COP area in women are significantly smaller than in men $(P<0.05)$. Women have better real-time balance control ability than men with smart insoles. Conclusions: The simple intelligent wearable assisted devices can immediately increase the control ability in static stance of men and women, and women have better real-time balance control ability than men.
\end{abstract}

Key words: gender difference, center of pressure, visual feedback, balance control

\section{Introduction}

The body maintains its stability through its perception of space (i.e., balance ability) [4] and balance control is one of the important physical fitness in humans. To maintain such a balance control in static body position, the body typically uses "hip strategy" or "ankle strategy" to project the center of mass (COM) in the base of support [3]. The COM sway and its spatial relationship with the base of support are the basis for balance control and performance [8]. Therefore, in the study of biomechanics, the change in the center of pressure (COP) in balance of systems is often considered as the main analysis parameter. The smaller the plantar COP amplitude, the higher the body's ability to maintain the balance control in static body position [24]. In other words, a good control of the change in plantar COP while maintaining the balance control in static body position is beneficial to the performance of balance ability. Previous studies have found that COP amplitude and mean velocity of patients with spinal

\footnotetext{
* Corresponding author: Chun-Sheng Ho, Lo-Hsu Medical Foundation, Inc., Lotung Poh-Ai Hospital, Luodong 265501, Taiwan. E-mail: cochonho@gmail.com

Received: December 3rd, 2020

Accepted for publication: February 5th, 2021
} 
cord injury decreased after visual feedback (VF) standing balance training, indicating that the ability of static and dynamic stability improved significantly after training [19]. After applying wearable devices to balance training for the elderly, the COP area and COP parameters displayed a significant decrease, indicating that balance training is effective for improving balance control and functional performance in older adults [21]. Furthermore, the single leg balance test results before exercise can identify people prone to sprain, and balance training may be able to prevent injury such as ankle sprain in exercise people [29]. The system has been reported as an effective balance test parameter [16]. Therefore, maintenance or improvement of balance is important for athletes, the elderly, those with ankle or knee injury history, and patients with sarcopenia.

Through the integration of vision, hearing, proprioception, and multiple sensory inputs from the central nervous system, organisms understand the relative movements of various parts of the body, the relationship between the body and the environment, and achieve the goal of stable standing by continuously adjusting muscle activity [1]. Regarding the distance between a space and an object, the prediction, direction, reaction time, time point, stability, and balance reflected by the brain are mainly provided by vision, and these factors significantly affect players' performances in competitions [28]. Therefore, the visual system is crucial for balance control. When moving, human beings often use the information provided by the visual system as a reference [26]. Real-time VF can effectively reduce COP sway and accelerate posture control during standing posture balance. Past studies used the Wii balance board real-time feedback function to improve postural control and balance in people with multiple disabilities [22]. VF can strengthen the body's control of COP displacement and reduce the movement of the body's COM, and finally achieve the rehabilitation effect of improving the body's balance [17]. Therefore, assisting proprioception through the balance board or VF system may improve body posture and stability during movement or balance control.

The body balance system can be affected by many different factors, including age, height, BMI, and gender [12]. Gender differences exist in human postural stability. Past studies comparing boys and girls of the same age indicated that girls often demonstrated less postural sway [7], indicating gender differences in maintaining body balance. Furthermore, the study of gender differences in children's postural stability revealed that girls can integrate sensory input more effectively and rely more on somatosensory feedback than boys [23]. In addition, men are more likely to sway and cause physical instability than women because of their greater COP displacement during postural control [25]. Static and dynamic exercises show that women are excellent in maintaining to balance in standing position, affording posture stabilization and body balance maintenance [20]. Previous studies have shown that men have greater COP displacements after open-eyes/closed-eyes balance training, indicating that men are more unstable than women [2]. Therefore, there can have gender differences on balance control ability, and women may have better balance.

Smart wearable devices have been vigorously used in daily life. In the test of muscle fatigue in young healthy people, the vibration of insoles can compensate for the postural instability caused by fatigue and maintain the body balance [11]. Therefore, using realtime VF on the position of the COP provided by smart wearable devices effectively to maintain body balance can yield the benefits of technology-assisted homeostasis in maintaining body balance and help maintain body balance and physical rehabilitation. This study hypothesizes that women may be more capable to maintain a balanced posture than men and that additional visual feedback provided by smart wearable devices may have an effect on improving the quality of balance control. The aim of this study was to investigate the immediate effects of real-time VF information provided by smart insoles on the balance control ability in static body position movement.

\section{Materials and methods}

\section{Participants}

Forty healthy participants [20 males (age: 19.63 \pm 0.73 ; height: $1.67 \pm 0.04$; weight: $58 \mathrm{~kg} \pm 6$; mean \pm SD) and 20 females (age: $20.74 \pm 0.98$; height: 1.78 \pm 0.05 ; weight: $71 \mathrm{~kg} \pm 6$; mean $\pm \mathrm{SD}$ )] with no known balance pathology were included in the study. In addition, the inclusion criteria were normal visual acuity and contrast sensitivity. As required by the Declaration of Helsinki, participants were informed of the content, process, and precautions of the study group in order to understand and be willing to cooperate fully with the experimenter to sign the consent form. The study was approved by the Research Ethics Committee of Hualien Tzu Chi Hospital, Buddhist Tzu Chi Medical Foundation (IRB109-053-B). 


\section{Procedures}

Prior to the experiment, the participants performed a 5-min warm-up run and a rested for $1 \mathrm{~min}$. The 40 participants completed test with eyes open under one-leg stance (OLS) with VF and NF: left-leg stance non-visual feedback (LLS-NF), left-leg stance visual feedback (LLS-VF), right-leg stance non-visual feedback (RLS-NF), and right-leg stance visual feedback (RLS-VF). During the balance control in static body position test, the participants were instructed to stand around the center above a force plate. The VF group used an iPad as an smart wearable device to achieve a stable body posture. The Podoon APP displayed the dynamic point of the COP, and the participants maintained the dynamic point in the central circle as much as possible. The data for each condition were collected for $30 \mathrm{~s}$ and three times. The balance control in static body position movement included the support leg standing upright, and the nonsupport leg was flexed at the knee of the support leg with the toes drooping naturally, the arms on the chest, and the palms on the shoulders. The iPad Pro used (iPad Pro, including the smart insoles APP) should be located at an eye-level height and $1.0 \mathrm{~m}$ apart from the participants.

\section{Equipment}

A force plate (BTS P6000, BTS Bioengineering, Italy) was used to collect the COP kinematics data: the COP maximum mediolateral amplitude (COPML maximum amplitude: Retrieves the difference between the maximum and minimum mediolateral amplitude of COP in the position of pressure center), the COP maximum anteroposterior amplitude (COPAP maximum amplitude: Retrieves the difference between the maximum and minimum anteroposterior amplitude of COP in the position of pressure center) [9], the COP mediolateral velocity (COPML velocity: Retrieves the mediolateral average velocity of the COP) [29], the COP anteroposterior velocity (COPAP velocity: Retrieves the anteroposterior average velocity of the COP), the COP radius (Retrieves the distance between the pressure center position coordinates and the average movement distance of the COP), and COP area (The COP area was defined as the area of the circle whose radius was the average of all the radial distances of the COP from the average position of the COP). The COP change is an important index of balance control, the COP radius and COP area reveal a high correlation in the balance control parameters [14]. These parameters can be used to evaluate balance control. Force plate signals were collected at a sampling frequency of $600 \mathrm{~Hz}$. Each data point of the pre-test and post-test was calculated from the average of three COPs.

\section{Statistical analysis}

Statistical analyses were performed using MATLAB (R2014a, The MathWorks, USA). A mixed-design two--way ANOVA was performed and included the following: two vision conditions (NF and VF) $\times$ gender (woman and man) were used to compare the $\mathrm{COP}_{\mathrm{ML}} / \mathrm{COP}_{\mathrm{AP}}$ maximum amplitude, $\mathrm{COP}_{\mathrm{ML}} / \mathrm{COP}_{\mathrm{AP}}$ velocity $\mathrm{COP}$ radius, and $\mathrm{COP}$ area. If significant interactions existed, the simple main effect post hoc test was used to assess differences between gender at two visual conditions. If no significant interaction existed, main effects were assessed. Sphericity was analyzed by Mauchly's test, followed by a Greenhouse-Geisser correction when necessary. The statistical significance level was set at $\alpha=0.05$. Intraclass correlation coefficients (ICC) were calculated for the COP parameters in the NF and VF, as poor ( ICC $<0.40)$, moderate $(0.40 \leq \mathrm{ICC}<0.60)$, good $(0.60 \leq \mathrm{ICC}<0.80)$, and excellent (ICC $\geq 0.80$ ).

\section{Results}

\section{Gender differences and vision conditions in $C O P_{M L}$ and $C O P_{A P}$ maximum amplitude parameter analysis}

The results of the gender differences and vision conditions in $\mathrm{COP}_{\mathrm{ML}}$ and $\mathrm{COP}_{\mathrm{AP}}$ maximum amplitude parameter are shown in Fig. 1. The $\mathrm{COP}_{\mathrm{ML}}$ maximum amplitude differed significantly in the vision conditions $(p<0.001)$; the analysis of vision conditions indicated a significant decrease in RLS-VF-Man/Woman compared with RLS-NF-Man/Woman $(\nabla 9.56 \%$ and $\nabla 15.60 \%)$. The main effect of the $\mathrm{COP}_{\mathrm{AP}}$ maximum amplitude differed significantly by gender $(p=0.013)$; the analysis of gender indicated a significant decrease in RLS-VF/NF-Woman compared with RLS-VF/NF-Man $(\nabla 16.17 \%$ and $\nabla 22.42 \%)$. The vision conditions differed significantly $(p<0.001)$, the analysis of vision conditions indicated a significant decrease in RLS-VF-Man/Woman compared with RLS-NF-Man/Woman $(\nabla 22.24 \%$ and $\nabla 15.96 \%)$. The main effect of the $\mathrm{COP}_{\mathrm{ML}}$ maximum amplitude was a significant difference in the vision conditions $(p<0.001)$; the analysis of vision conditions indicated a significant decrease in LLS-VF-Man/Woman compared with LLS-NF-Man/Woman $(\nabla 14.28 \%$ and $\nabla 9.93 \%)$. The main effect of the $\mathrm{COP}_{\mathrm{AP}}$ maximum amplitude differed significantly by gender 

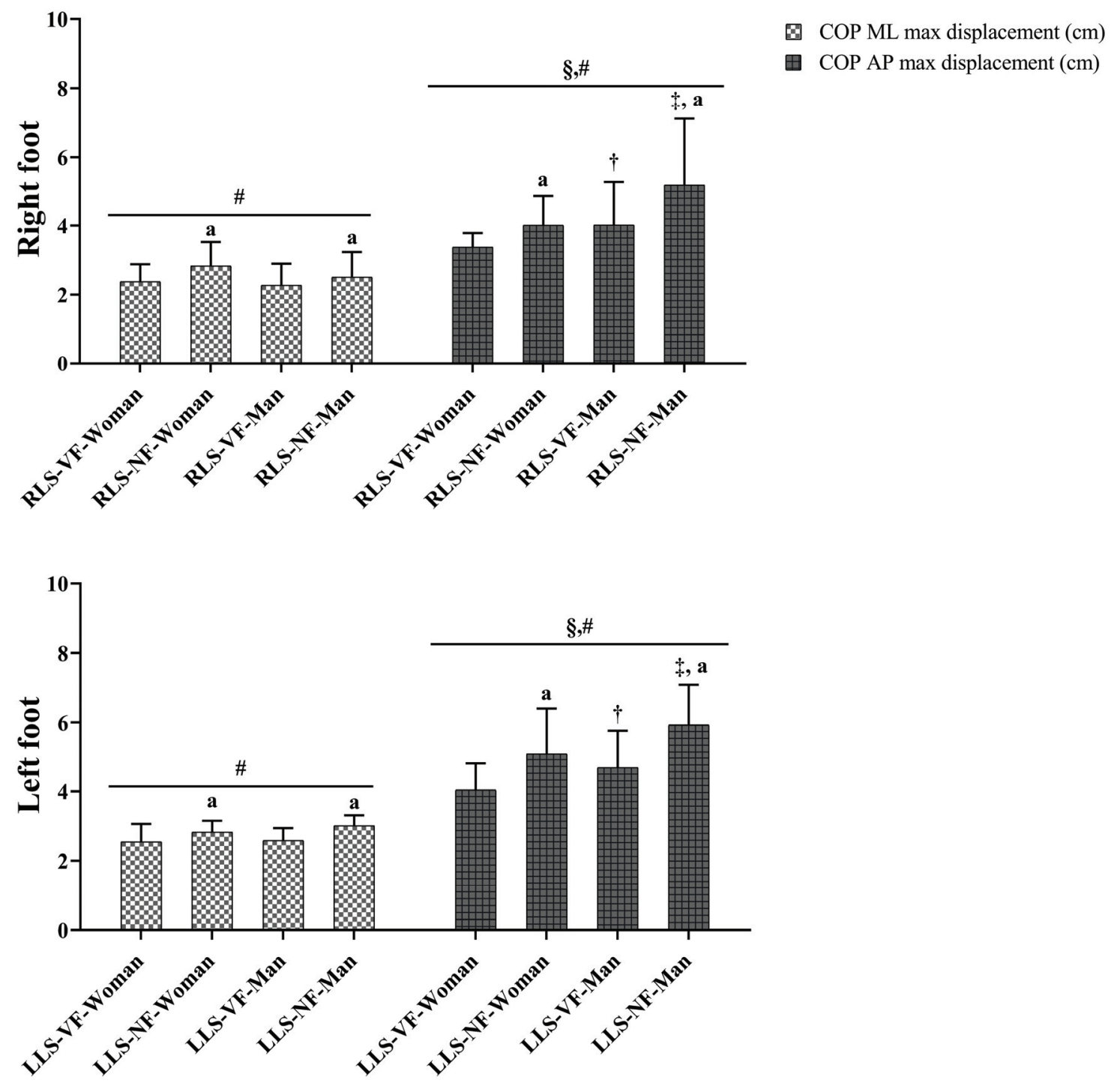

Fig. 1. Gender differences and vision conditions in COPML and COPAL maximum displacement parameter.

* Indicates significant difference in interaction $(p<0.05)$. Indicates significant difference in main effect (gender) $(p<0.05)$. \# indicates significant difference in main effect (vision conditions) $(p<0.05) .{ }^{a}$ indicates significant difference from visual test. $\dagger$ indicates significant difference in man and woman's visual test. $\ddagger$ indicates significant difference in men and women's non-visual test.

Interaction (vision conditions $\times$ gender) of the COPML maximum displacement or COPAP maximum displacement in RLS/LLS did not differ significantly $(p>0.05)$ (men: $N=20$, women: $N=20)$

$(p=0.007)$; the analysis of gender showed a significant decrease in LLS-VF/NF-Woman compared with LLS-VF/NF-Man ( $\nabla 13.67 \%$ and $\nabla 13.40 \%)$; the vision conditions showed a significant difference $(p<0.001)$; the analysis of vision conditions showed a significant decrease in LLS-VF-Man/Woman compared with LLS-NF-Man/Woman ( $\nabla 21.80 \%$ and $\nabla 20.78 \%$ ). Therefore, the $\mathrm{COP}_{\mathrm{ML}}$ maximum amplitude or $\mathrm{COP}_{\mathrm{AP}}$ maximum amplitude of men/women decreased significantly in the VF group compared with the NF group, whereas the $\mathrm{COP}_{\mathrm{AP}}$ maximum amplitude of women was significantly lower than that of men in each balance condition. The $\mathrm{COP}_{\mathrm{ML}}$ and $\mathrm{COP}_{\mathrm{AP}}$ maximum amplitude ICC ranged from 0.75 to 0.84 .

\section{Gender differences and vision conditions} in $C O P_{M L}$ and $C O P_{A P}$ velocity parameter analysis

The results of the gender differences and vision conditions in $\mathrm{COP}_{\mathrm{ML}}$ and $\mathrm{COP}_{\mathrm{AP}}$ velocity parameter are shown in Fig. 2. The main effect of the $\mathrm{COP}_{\mathrm{ML}}$ velocity had a significant difference in the vision conditions $(p=0.001)$; the analysis of vision conditions showed a significant decrease in RLS-VF-Man/Woman compared to RLS-NF-Man/Woman $(\nabla 13.43 \%$ and $\nabla 12.43 \%)$. The main effect of the $\mathrm{COP}_{\mathrm{AP}}$ velocity was a significant difference in gender $(p<0.001)$; the analysis of gender showed a significant decrease in RLS-VF/NF-Woman compared to RLS-VF/NF-Man 


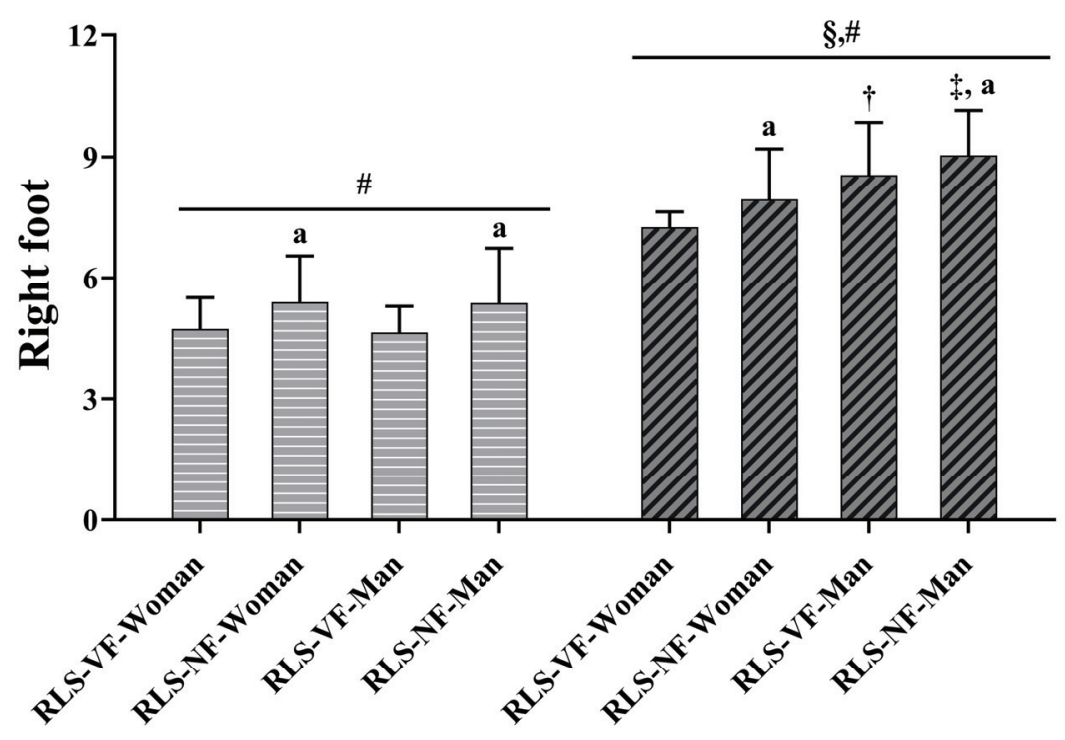

$\begin{array}{ll}\boxminus & \text { COP ML velocity }(\mathrm{cm} / \mathrm{s}) \\ \square & \text { COP AP velocity }(\mathrm{cm} / \mathrm{s})\end{array}$

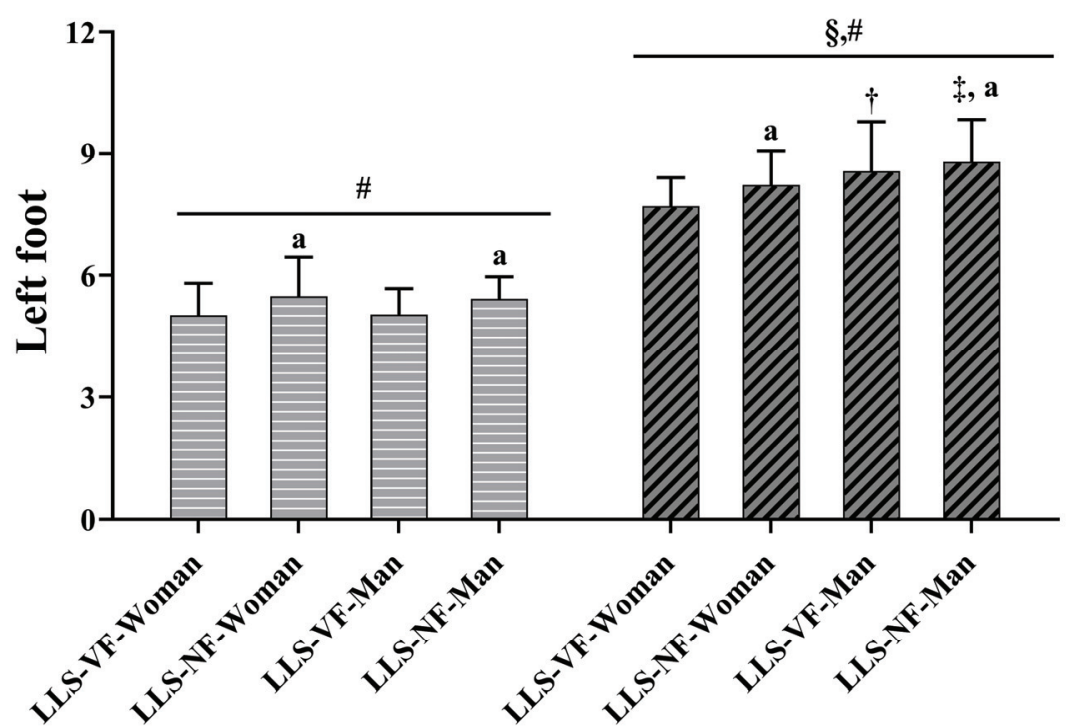

Fig. 2. Gender differences and vision conditions in COPML and COPAL velocity parameter.

* Indicates significant difference in interaction $(p<0.05)$. Indicates significant difference in main effect (gender) $(p<0.05)$.

\# indicates significant difference in main effect (vision conditions) $(p<0.05) .{ }^{a}$ indicates significant difference from visual test. $\dagger$ indicates significant difference in man and woman's visual test. $\ddagger$ indicates significant difference in men and women's non-visual test. Interaction (vision conditions $\times$ gender) of the COPML maximum displacement or COPAP maximum displacement in RLS/LLS did not differ significantly $(p>0.05)(\operatorname{man}: N=20$, woman: $N=20)$

$(\nabla 15.20 \%$ and $\nabla 22.44 \%)$. The vision conditions differed significantly $(p=0.001)$; the analysis of vision conditions showed a significant decrease in RLS-VF-Man/Woman compared with RLS-NF-Man/Woman $(\nabla 5.42 \%$ and $\nabla 8.69 \%)$. The main effect of the $\mathrm{COP}_{\mathrm{ML}}$ velocity had a significant difference in vision conditions $(p=0.001)$, analysis of vision conditions had a significant decrease in LLS-VF-Man/Woman compared to LLS-NF-Man/Woman ( $\nabla 7.18 \%$ and $\nabla 8.39 \%)$. The main effect of the $\mathrm{COP}_{\mathrm{AP}}$ velocity had a significant difference in gender $(p=0.020)$; the analysis of gender showed a significant decrease in LLS-VF/NF-
-Woman compared with LLS-VF/NF- Man ( $\nabla 10.06 \%$ and $\nabla 6.49 \%$ ). The vision conditions differed significantly $(p<0.001)$; the analysis of vision conditions showed a significant decrease in LLS-VF-Man/ Woman compared to LLS-NF-Man/Woman ( $\nabla 2.62 \%$ and $\nabla 6.82 \%$ ). Therefore, the $\mathrm{COP}_{\mathrm{ML}}$ velocity or $\mathrm{COP}_{\mathrm{AP}}$ velocity of men/women decreased significantly decreased in the VF group compared to the NF group, and the $\mathrm{COP}_{\mathrm{AP}}$ velocity of women was significantly lower than that of men in each balance condition. This shows that women have better balance control ability than men. The $\mathrm{COP}_{\mathrm{ML}}$ and $\mathrm{COP}_{\mathrm{AP}}$ velocity $\mathrm{ICC}>0.90$. 
Gender differences and vision conditions

in $C O P$ radius and COP area parameter analysis

The results of the gender differences and vision conditions in COP radius and COP area parameter are shown in Fig. 3. The main effect of the COP radius had a significant difference in gender $(p=0.021)$; the analysis of gender showed a significant decrease in RLS-VF/NF-Woman compared to RLS-VF/NF-Man $(\nabla 14.10 \%$ and $\nabla 17.78 \%)$. The vision conditions differed significantly $(p=0.001)$; the analysis of vision conditions showed a significant decrease in RLS-VF-Man/Woman compared with RLS-NF-Man/Woman $(\nabla 13.33 \%$ and $\nabla 21.80 \%)$. The main effect of the COP area had a significant difference in gender $(p=0.001)$; the analysis of gender showed a significant decrease in RLS-VF/NF-Woman compared to RLS-VF/NF-Man $(\nabla 30.16 \%$ and $\nabla 33.44 \%)$. The vision conditions
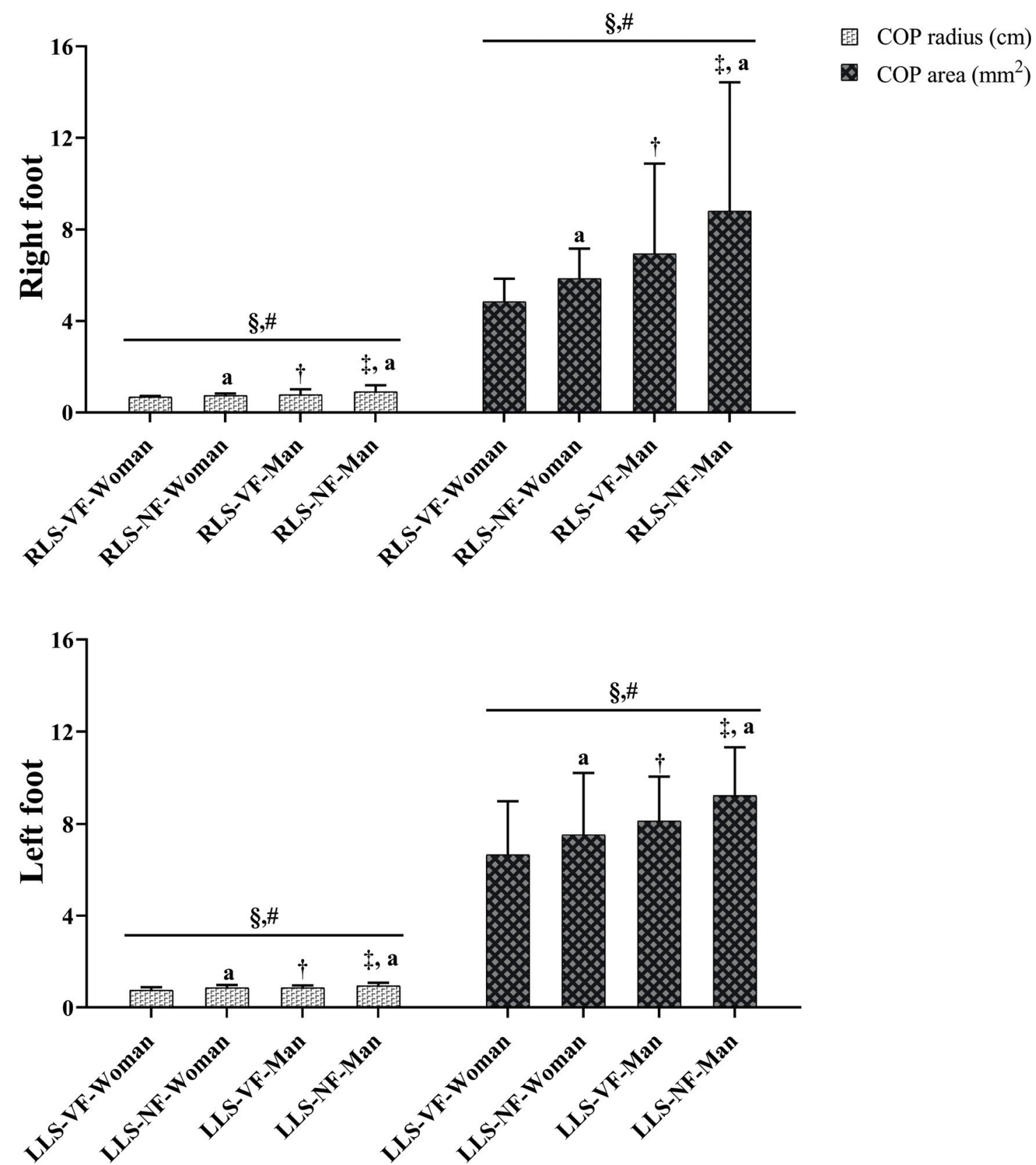

Fig. 3. Gender differences and vision conditions in COP radius and COP area parameter.

* Indicates significant difference in interaction $(p<0.05)$. $§$ Indicates significant difference in main effect (gender) $(p<0.05)$. \# indicates significant difference in main effect (vision conditions) $(p<0.05){ }^{a}$ indicates significant difference from visual test. $\dagger$ indicates significant difference in man and woman's visual test. $\ddagger$ indicates significant difference in men and women's non-visual test. Interaction (vision conditions $\times$ gender) of the COPML maximum displacement or COPAP maximum displacement in RLS/LLS did not differ significantly $(p>0.05)$ (men: $N=20$, women: $N=20)$ 
differed significantly $(p=0.001)$; the analysis of vision conditions showed a significant decrease in RLSVF-Man/Woman compared to RLS-NF-Man/Woman $(\nabla 21.16 \%$ and $\nabla 16.84 \%)$. The main effect of the COP radius had a significant difference in gender $(p=0.003)$; the analysis of gender showed a significant decrease in LLS-VF/NF-Woman compared to LLS-VF/NF-Man $(\nabla 11.49 \%$ and $\nabla 9.37 \%)$. The vision conditions differed significantly $(p<0.001)$; the analysis of vision conditions showed a significant decrease in LLS-VF-Man/Woman compared to LLS-NF-Man/Woman $(\nabla 9.37 \%$ and $\nabla 11.49 \%)$. The main effect of the COP area had a significant difference in gender $(p=0.020)$; the analysis of gender showed a significant decrease in LLS-VF/NF-Woman compared with LLS-VF/NF-Man $(\nabla 18.22 \%$ and $\nabla 18.32 \%)$. The vision conditions differed significantly $(p=0.002)$; the analysis of vision conditions showed a significant decrease in LLS-VF-Man/Woman compared to LLS-NF-Man/Woman $(\nabla 11.93 \%$ and $\nabla 14.21 \%)$. Therefore, the COP radius or COP area of men/women decreased significantly in the VF group compared to the NF group, and the COP radius and COP area of women decreased significantly decreased compared to that of men in each balance condition. The COP radius and COP area ICC ranged from 0.64 to 0.88 .

\section{Discussion}

The purpose of this study was to investigate the immediate effects of real-time visual feedback information provided by smart insoles on the balance control in static body position movement for healthy young people of different genders. The results showed that the amplitude, velocity, radius, and area of COP decreased significantly in men or women in OLS-VF and the $\mathrm{COP}_{\mathrm{AP}}$ amplitude, $\mathrm{COP}_{\mathrm{AP}}$ velocity, $\mathrm{COP}$ radius, and COP area of women has decreased significantly in men. Furthermore, the COP parameter decreased as the body stability strengthened [24]. The VF real-time of the balance control in static body position ability provided by smart insoles was higher than that of NF, and the real-time balance control ability of women was higher than that of men.

After VF was provided, the $\mathrm{COP}_{\mathrm{AP}}$ maximum amplitude, and $\mathrm{COP}_{\mathrm{ML}}$ maximum amplitude reduced, indicating the effectiveness of VF as a posture control strategy. The direction of the COP track shows different control strategies for postural sway during standing. The postural sway in the mediolateral (ML) direction is related to hip joint stability, whereas the postural sway in the anteroposterior (AP) direction is related to ankle joint stability [10]. In the AP direction, body sway and posture changes are closely related to the ankle neuromuscular function. The "ankle strategy" can increase the stability of the ankle joint and reduce the COP amplitude to improve the balance ability [13]. In this study, the decrease in COP amplitude in the AP direction may be caused by the effect of VF on the stability of the ankle joint. Balance control in static body position can be maintained through an active torque regulation/control of sensory motor integration for muscle contraction during ankle position modulation [28]. Therefore, the intervention of VF enables the COP to be displayed on the screen, and the body actively controls the COP according to the change in the COP to maintain physical stability. The central nervous system is fully involved in regulating the movement of the ankle muscles to maintain the stability of the COM. In this study, the $\mathrm{COP}_{\mathrm{ML}}$ maximum amplitude decreased after VF intervention. Previous studies revealed that the COP amplitude improved in both the and AP directions during the exercise of stable of the balance control in static body position [5]. When controlling the ML amplitude, the "hip strategy" accompanied by VF control balance results in autonomous adduction and abduction [30]. Moreover, the amplitude in the ML direction can improve balance ability through this autonomous adduction and abduction change. Therefore, the balance control in static body position standing after VF may cause the joint activity of "ankle strategy" and "hip strategy" to maintain stability, thereby reducing the degree of body sway and amplitude variation after VF.

The $\mathrm{COP}_{\mathrm{AP}}$ velocity, and $\mathrm{COP}_{\mathrm{ML}}$ velocity decreased after VF intervention. Previous studies regarding the balance of soccer players revealed that the decrease in COP velocity was caused by more effective prediction of body position changes by the visual system of soccer players [28]. The difference in parameters before and after the experiment in this study may be caused by the VF information obtained after VF intervention to predict body position changes. The decrease in the $\mathrm{COP}_{\mathrm{AP}}$ velocity may result from the body's assessment of body status through VF to achieve better stability [28]. When standing, the body feeds back position and sensory information to the central nervous system to control the muscle activity of the ankle joint mechanism and facilitate the body in maintaining balance. In the OLS, the $\mathrm{COP}_{\mathrm{AP}}$ velocity controlled by the "ankle strategy" and the $\mathrm{COP}_{\mathrm{ML}}$ velocity controlled by the "hip strategy" decreased, thereby increasing the stability of balance control in standing position [6]. The maintenance of the balance control in static body 
position in the human body is typically completed by the activities of the trunk and lower limb muscles, such as the "hip strategy" or "ankle strategy" [3]. Therefore, the decrease in the $\mathrm{COP}_{\mathrm{AP}}$ velocity and $\mathrm{COP}_{\mathrm{ML}}$ velocity may be the result of the mediation of joint motion between the ankle and hip after VF, which can enhance the stability of the lower extremities.

The COP area and COP radius under VF decreased, indicating that the stability of the participants may increase after VF intervention. Previous studies revealed that using $\mathrm{VF}$ and virtual reality balance tests can reduce the COP radius and area [15]. Therefore, the enhancement of VF strengthens the participants' ability in controlling balance. Balance control is a dynamic adaptation process of active (autonomous control) and passive (accidental, external disturbance) balance control actions. When visual information increases, the body's autonomous control ability strengthens. Moreover, the enhancement in autonomous control ability reduces the amplitude and COP radius to strengthen the physical stability [26]. The decrease in COP area and COP radius in this study may be caused by the increase in the autonomous control ability of VF for enhancing physical stability. In addition, previous studies revealed that healthy adults under VF maintained body balance more effectively [18]. Therefore, visual information strengthens the sensory system of young people and increases the ability of autonomous control under the regulation of the central nervous system to enhance physical stability.

Previous studies revealed that the COP amplitude of men was greater than that of women [2], which is consistent with the results of this study, i.e., the $\mathrm{COP}_{\mathrm{AP}}$ amplitude of men is greater than that of women. The sway lengths of men and women were longer in the AP direction than in the ML direction, and the amplitude range of men was larger; women indicated a stronger ability to perceive surrounding information and effectively integrated perceptual information [27]. In addition, the difference in height and weight between men and women caused the amplitude range of men to be greater than that of women [2]. Therefore, in this study, women used the surrounding sensory system to integrate movement and promote greater stability. The $\mathrm{COP}_{\mathrm{AP}}$ velocity of men in this study was greater than that of women, which may be caused by the higher upper body weight of men, resulting in a higher relative vertical position of the COM [2]. This affects the projection of the COM and reduces the physical stability. In the absence of sensory aids, the posture control ability of men was worse [25]. Therefore, the lower COM of women with the participation of intelligent auxiliary equipment is beneficial for controlling the stability of the body. The COP radius and COP area of both women and men decreased after VF intervention, whereas those of women decreased more than those of men. Compared to men, women have less motor function, are more sensitive to surrounding information, and exhibit higher autonomous ability in balance control [25]. Therefore, smart insoles can increase VF and maintain physical stability. During balance control, women use the surrounding sensory information to increase autonomous control under the regulation of the central nervous system, thereby improving the stability of static standing. The limitations of this study were that the EMG and kinematic images of participants were not collected; moreover, it was difficult to detect the balance regulation of joint muscle vision conditions and joint angle. Therefore, the application of smart wearables in several weeks of balance training to assist the body to improve balance should be applied to individuals with unstable postures in future studies to investigate the benefits of VF on balance improvement.

The limitation of this study was that there was no comparison of age balance performance differences. Maturational processes also affecting the development of balance performance in youth differ in onset and velocity inter individually, consequently complicating comparisons based on chronological age only. Future studies on children and adolescents of different ages should be compared in terms of their physiological age balance to explore their athletic performance.

\section{Conclusions}

Intelligent auxiliary equipment increases the participants' visual information, and the increase in visual feedback may promote the integration of the central nervous system to increase the body's real-time postural stability. When applied to visual feedback to increase visual information, a smart auxiliary equipment can increase the autonomous control ability of young people. It is beneficial for the central nervous system to control the balance mechanism to perform autonomous adjustments to maintain physical stability, and it might be better for women to control the sensory information around them autonomously. In this study, a simple smart auxiliary equipment was used to provide visual feedback information of the COM to maintain the real-time balance ability of healthy people of different genders for static standing. Women demonstrated higher real-time balance ability in visual feedback than men. Moreover, the use of smart insoles to 
provide visual feedback yielded intuitive, simple, and real-time feedback with strong operability. In the future, this technology can be further extended to various rehabilitation purposes or improve the balance of the human body.

\section{Funding}

This research received grant from the Lo-Hsu Medical Foundation, Inc., Lotung Poh-Ai Hospital (Number: E157).

\section{Acknowledgments}

The authors thank Shi-Jie Xue and Yang Liu for conducting the biochemical examinations. The study was approved by the Research Ethics Committee of Hualien Tzu Chi Hospital, Buddhist Tzu Chi Medical Foundation (IRB109-053-B).

\section{References}

[1] Brauer S.G., Woollacott M., Shumway-Cook A., The interacting effects of cognitive demand and recovery of postural stability in balance-impaired elderly persons, J. Gerontol. A Biol. Sci. Med. Sci., 2001, 56, M489-M496.

[2] BRYANT E.C. et al., Gender differences in balance performance at the time of retirement, Clin. Biomech. (Bristol, Avon), 2005, 20, 330-335.

[3] Horak F., Kuo A., Postural adaptation for altered environments, tasks, and intentions, in Biomechanics and neural control of posture and movement, Springer, 2000, 267-281.

[4] Hrysomallis C., Balance ability and athletic performance, Sports Med., 2011, 41, 221-232.

[5] KaJi A. et al., Transient effect of core stability exercises on postural sway during quiet standing, J. Strength Cond. Res., 2010, 24, 382-388.

[6] Kоуама K., Yamauchi J., Altered postural sway following fatiguing foot muscle exercises, PLoS One, 2017, 12, e0189184.

[7] LEE A.J., LIN W.-H., The influence of gender and somatotype on single-leg upright standing postural stability in children, J. Appl. Biomech., 2007, 23, 173-179.

[8] LizAMA L.E.C. et al., Centre of pressure or centre of mass feedback in mediolateral balance assessment, J. Biomech., 2015, 48, 539-543.

[9] MochizuKi G. et al., Low-frequency common modulation of soleus motor unit discharge is enhanced during postural control in humans, Experimental Brain Research, 2006, 175, 584-595.

[10] MochizUKi G. et al., Low-frequency common modulation of soleus motor unit discharge is enhanced during postural control in humans, Exp. Brain Res., 2006, 175, 584-595.

[11] Moon J. et al., Shoes with active insoles mitigate declines in balance after fatigue, Sci. Rep., 2020, 10, 1951-1951.
[12] OlchowiK G. et al., The human balance system and gender, Acta Bioeng. Biomech., 2015, 17.

[13] PARK K.H., LIM J.Y., KIM T.H., The effects of ankle strategy exercises on unstable surfaces on dynamic balance and changes in the COP, J. Phys. Ther. Sci., 2016, 28, 456-459.

[14] Ravi D., Kumar N., Singhi P., Effectiveness of virtual reality rehabilitation for children and adolescents with cerebral palsy: an updated evidence-based systematic review, Physiotherapy, 2017, 103, 245-258.

[15] RAVI D.K., KUMAR N., SINGHI P., Effectiveness of virtual reality rehabilitation for children and adolescents with cerebral palsy: an updated evidence-based systematic review, Physiotherapy, 2017, 103, 245-258.

[16] Rотн A.E. et al., Comparisons of static and dynamic balance following training in aquatic and land environments, Journal of Sport Rehabilitation, 2006, 15, 299-311.

[17] Rougier P., FARENC I., Berger L., Modifying the gain of the visual feedback affects undisturbed upright stance control, Clin. Biomech., 2004, 19, 858-867.

[18] S B. and PR R., Relation between postural control assessment with eyes open and centre of pressure visual feedback effects in healthy individuals, Exp. Brain Res., 2009, 195, 145-152.

[19] SayenKo D.G. et al., Positive effect of balance training with visual feedback on standing balance abilities in people with incomplete spinal cord injury, Spinal Cord., 2010, 48, 886-893.

[20] Schedler S., Kiss R., Muehlbauer T., Age and sex differences in human balance performance from 6-18 years of age: A systematic review and meta-analysis, PLoS one, 2019, 14, e0214434.

[21] SCHWENK M. et al., Interactive balance training integrating sensor-based visual feedback of movement performance: a pilot study in older adults, J. Neuroeng. Rehabilitation, 2014, 11, 164.

[22] SHIH C.-H., SHIH C.-T., Chiang M.-S., A new standing posture detector to enable people with multiple disabilities to control environmental stimulation by changing their standing posture through a commercial Wii Balance Board, Res. Dev. Disabil., 2010, 31, 281-286.

[23] Smith A., Ulmer F., Wong D., Gender differences in postural stability among children, J. Hum. Kinet., 2012, 33, 25-32.

[24] Stanković M., RAdenKović O., The status of balanve in preschool children involved in dance program, Research in Kinesiology, 2012, 40.

[25] STEINDL R. et al., Effect of age and sex on maturation of sensory systems and balance control, Dev. Med. Child Neurol., 2006, 48, 477-482.

[26] STINS J. et al., Sway regularity reflects attentional involvement in postural control: Effects of expertise, vision and cognition, Gait \& Posture, 2009, 30, 106-109.

[27] Sullivan E.V. et al., Postural sway reduction in aging men and women: relation to brain structure, cognitive status, and stabilizing factors, Neurobiol. Aging, 2009, 30, 793-807.

[28] ThOMPson L.A. et al., Balance Performance as Observed by Center-of-Pressure Parameter Characteristics in Male Soccer Athletes and Non-Athletes, Sports (Basel, Switzerland), 2017, 5.

[29] Trojian T.H., McKeag D.B.J.B.j.o.s.m., Single leg balance test to identify risk of ankle sprains, 2006, 40, 610-613.

[30] WINTER D.A. et al., Unified theory regarding $A / P$ and $M / L$ balance in quiet stance, J. Neurophysiol., 1996, 75, 2334-2343. 